Supporting Information for

\title{
Electrochemiluminescence Immunosensor for the Detection of \\ Carcinoembryonic Antigen based on Oxygen Vacancy-Rich Co3O4 \\ Nanorods and Luminol
}

Yun Tang, Bihong Zhang, Yuan Wang, Faqiong Zhao, Baizhao Zeng*

College of Chemistry and Molecular Sciences, Wuhan University, Wuhan 430072, Hubei Province, PR China

\section{EXPERIMENTAL SECTION}

\subsection{Reagents and apparatus}

Cobalt nitrate hexahydrate $\left(\mathrm{Co}\left(\mathrm{NO}_{3}\right)_{2} \cdot 6 \mathrm{H}_{2} \mathrm{O}\right)$, urea, and luminol have been acquired from Aladdin Chemistry Co., Ltd. (Shanghai, China). Aniline ( $\geq 98 \%)$, thioglycolic acid (TGA), $\mathrm{NaBH}_{4}, \mathrm{Na}_{2} \mathrm{HPO}_{4} \cdot 12 \mathrm{H}_{2} \mathrm{O}$, and $\mathrm{NaH}_{2} \mathrm{PO}_{4} \cdot 2 \mathrm{H}_{2} \mathrm{O}$ were purchased from Sinopharm Chemical Reagent Co. Ltd., China. The tumor markers carcinoembryonic antigen (CEA, CEA McAb) were purchased from Linc-Bio Science Co. Ltd (Shanghai, China). 1-Ethyl-3-(3-dimethyl aminopropyl) carbodiimide hydrochloride (EDC) and bovine serum albumin (BSA) were obtained from Shanghai Chemical Reagent Co., Ltd. (China). The antibody, antigen solution, and washing solution were prepared with phosphate buffer solution (PBS, pH 7.4, 0.10 M). Normal human serum came from Shanghai Fusheng Industrial Co., Ltd. Other chemical reagents used were of analytical grade and their aqueous solutions were prepared with deionized water (DI water, $18.25 \mathrm{M} \Omega / \mathrm{cm}$ ).

Electrochemiluminescence and cyclic voltammetry (CV) measurements were conducted on a Model MPI-EII ECL analyzer (Xi'an Remex Instrument Co. Ltd., China) with a three-electrode system that consisted of a saturated $\mathrm{Ag} / \mathrm{AgCl}$ electrode as reference electrode, a modified glassy carbon electrode (GCE, $\Phi=3 \mathrm{~mm}$ ) as the working

\footnotetext{
${ }^{*}$ Corresponding author. Tel: 86-27-68752701, Fax: 86-27-68754067.

E-mail address: bzzeng@whu.edu.cn
} 
electrode and a platinum wire as the auxiliary electrode. The electrochemical impedance spectroscopy (EIS) experiment was implemented on a CHI660D electrochemical workstation (Shanghai Chenhua Instrument Co. Ltd., China). The scanning electron microscopy (SEM) experiment was performed using a Gemini300 (Oxford X-MAX); transmission electron microscopy (TEM) image was obtained using an FEI Tecnai G2 F2(OXFORD X-max 80T). X-ray diffraction (XRD) experiment was performed on a Bruker D8 diffractometer (Germany) with $\mathrm{Cu} \mathrm{K} \alpha$ radiation (40 KV, 40 $\mathrm{mA}$ ) over the $2 \theta$ range of $10-80^{\circ}$ at a scan rate of $10.0^{\circ} \mathrm{min}^{-1}$. X-ray photoelectron spectroscopy (XPS) analysis was carried out on an ESCALAB 250Xi spectrometer (Thermo Fisher Scientific Inc, America).

\subsection{Electrochemical measurements}

The electrochemical measurements had been carried out on a $\mathrm{CHI} 760 \mathrm{E}$ electrochemical workstation (CH Instruments, Chenhua Co., Shanghai, China) with a standard three-electrode system. Catalyst-coated glassy carbon electrode (diameter: 5 $\mathrm{mm}$ ), graphite rod, and $\mathrm{Hg} / \mathrm{HgO}$ electrode (MOE) (in 0.10 M PBS) served as a working electrode, counter electrode, and reference electrode, respectively. Before OER tests, the ink was prepared by dispersing $5.0 \mathrm{mg}$ catalyst into $1 \mathrm{~mL}$ solvent (i.e. isopropanol - 5\% Nafion with V/V of 49:1) with the assistant of sonication. Then, $6 \mu \mathrm{L}$ ink was carefully dropped on a GCE and dried. The supporting electrolyte (0.10 M pH 7.5 PBS) was air-saturated and the scan rate was $10 \mathrm{mV} / \mathrm{s}$, with a rotation speed of $1600 \mathrm{rpm}$. Linear sweep voltammogram (LSV) compared with the initial curve was conducted at the $1000^{\text {th }}$ cycle at a scan rate of $10 \mathrm{mV} / \mathrm{s}$ in the potential range of $0 \mathrm{~V}-+0.7 \mathrm{~V}$. The loading amount of $\mathrm{r}-\mathrm{Co}_{3} \mathrm{O}_{4} \mathrm{NRs}$ was around $30 \mu \mathrm{g} \mathrm{cm}_{\text {disc }}{ }^{-2}$. 


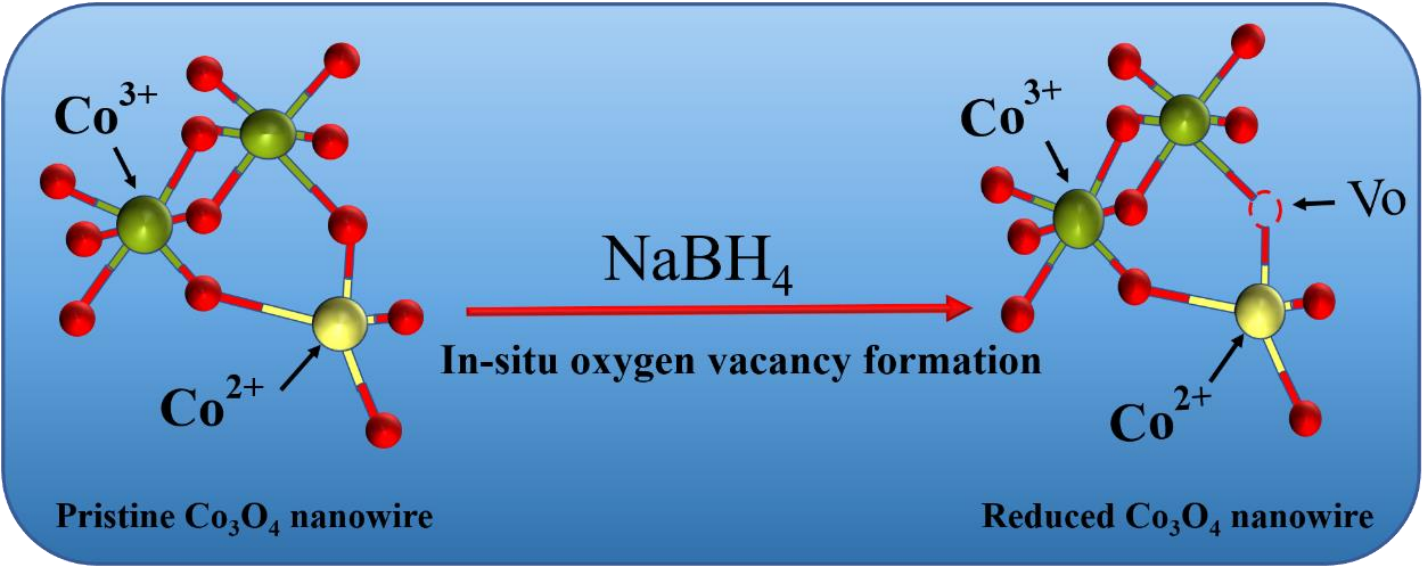

Figure S1. Schematic of in situ creation of oxygen vacancies in $\mathrm{Co}_{3} \mathrm{O}_{4} \mathrm{NWs}$ caused by $\mathrm{NaBH}_{4}$ reduction.

The pristine $\mathrm{Co}_{3} \mathrm{O}_{4} \mathrm{NWs}$ were treated with $\mathrm{NaBH}_{4}$ solution, oxygen vacancy occurred. ${ }^{1}$

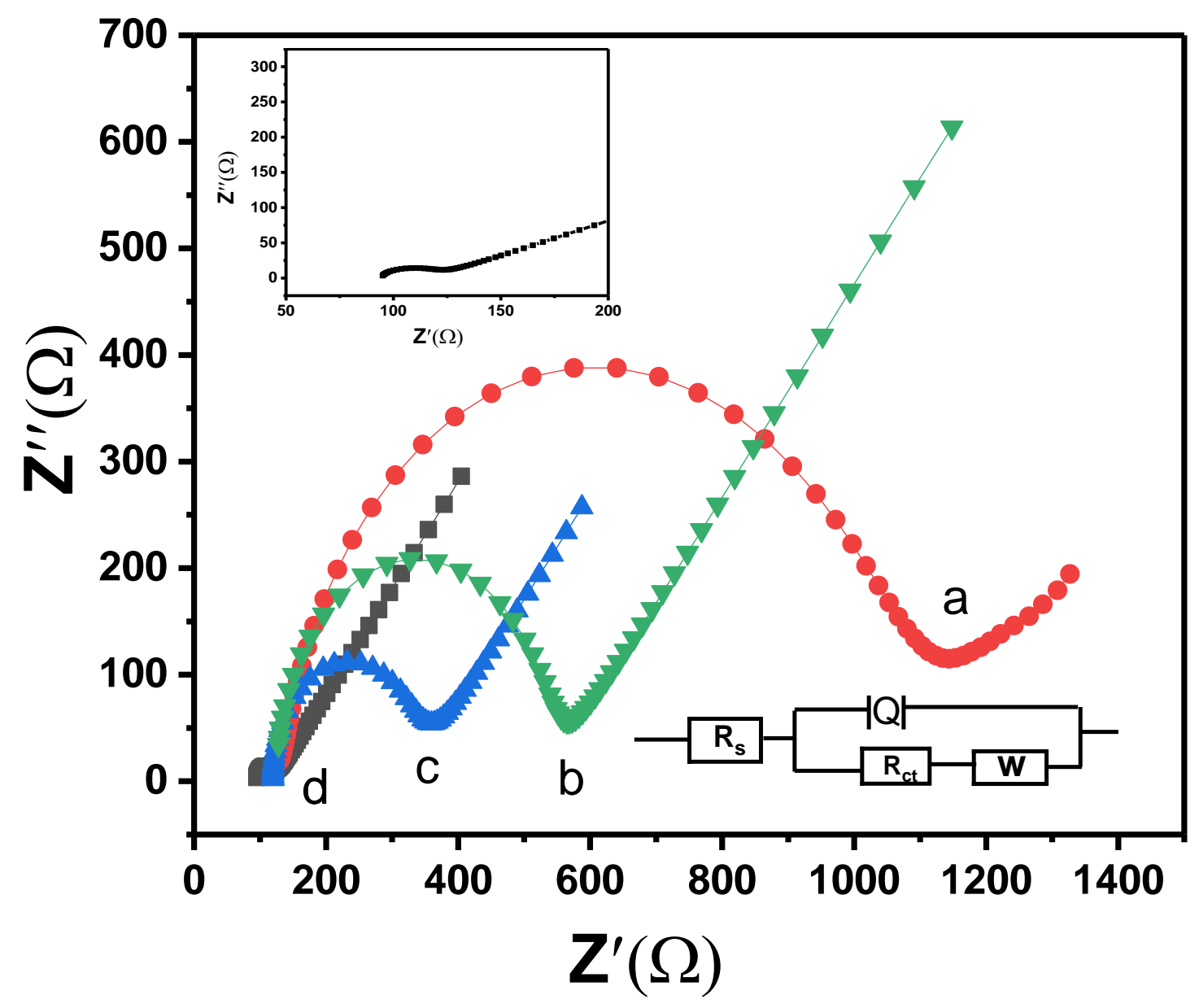

Figure S2. Electrochemical impedance spectra of different electrodes in $0.10 \mathrm{M} \mathrm{KCl}$ solution containing $5.0 \mathrm{mM}\left[\mathrm{Fe}(\mathrm{CN})_{6}\right]^{3-/ 4-}$. (a) Bare GCE, (b) p- $\mathrm{Co}_{3} \mathrm{O}_{4} / \mathrm{GCE}$ NWs. (c) 
$\mathrm{r}-\mathrm{Co}_{3} \mathrm{O}_{4} \mathrm{NWs} / \mathrm{GCE},(\mathrm{d})$ Luminol/GCE.

The electrochemical impedance spectra (EIS) of different electrodes (i.e. GCE, Luminol/GCE, $\mathrm{p}-\mathrm{Co}_{3} \mathrm{O}_{4} \mathrm{NWs} / \mathrm{GCE}$, and $\mathrm{r}-\mathrm{Co}_{3} \mathrm{O}_{4} \mathrm{NWs} / \mathrm{GCE}$ ) were recorded to assess the charge transfer resistance of different materials. ${ }^{2}$
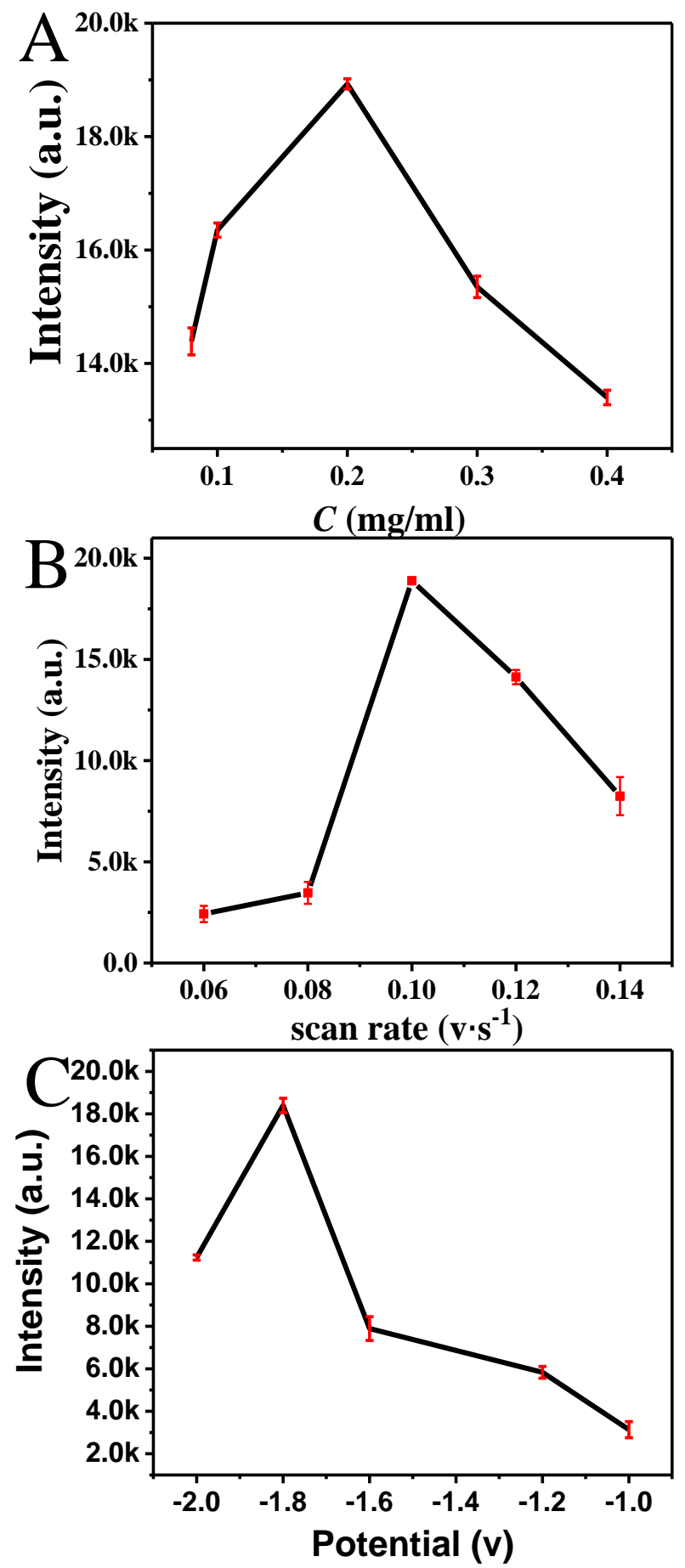
Figure S3. (A) Influence of scan rate $((0.06-0.14 \mathrm{~V} / \mathrm{s}))$ on ECL intensity. (B) Influence of $\mathrm{r}-\mathrm{Co}_{3} \mathrm{O}_{4} \mathrm{NWs}$ concentration $(0.08-0.4 \mathrm{mg} / \mathrm{mL})$ on ECL intensity. (C) Influence of initial potential $(-2.0--1.0 \mathrm{~V})$ on ECL intensity.

The experimental conditions of scan rate, $\mathrm{r}-\mathrm{Co}_{3} \mathrm{O}_{4} \mathrm{NWs}$ concentration, and initial potential were optimized for the ECL detection of CEA with r-Co $\mathrm{Co}_{3} \mathrm{O}_{4}$ NWs/luminol/GCE. ${ }^{3}$

\section{Reference}

1. Zhuang, L.; Ge, L.; Yang, Y.; Li, M.; Jia, Y.; Yao, X.; Zhu, Z. Ultrathin Iron-Cobalt Oxide Nanosheets with Abundant Oxygen Vacancies for the Oxygen Evolution Reaction. Adv. Mater. 2017.29. 1606793

2. Yan, K.; Shang, X.; Liu, Z.; Dong, B.; Lu, S; Chi, J.; Gao, W.; Chai, Y; Liu, C. A facile method for reduced $\mathrm{CoFe} 2 \mathrm{O} 4$ nanosheets with rich oxygen vacancies for efficient oxygen evolution reaction. Int. J. Hydrogen Energy. 2017. 42. 24150.

3. Liang, J.; Xu, Q.; Teng, Xu.; Guan, W.; Lu, C. Superoxide-Triggered Luminol Electrochemiluminescence for Detection of Oxygen Vacancy in Oxides. Anal. Chem. 2020. 92. 1628. 